\title{
Integração financeira internacional e crescimento econômico: uma crítica à abordagem convencional ${ }^{1}$
}

\author{
Aderbal Oliveira Damasceno ${ }^{2}$
}

\section{Resumo}

A defesa de um desenho institucional para as economias domésticas que permita a livre circulação de capitais entre as fronteiras é fundamentada por modelos teóricos que sublinham um conjunto de canais por meio dos quais a Integração Financeira Internacional levaria à melhoria na eficiência da alocação de recursos, estimulando o crescimento econômico de longo prazo dos países. O objetivo do trabalho é realizar uma crítica a essa abordagem, mostrando as fragilidades das teorias que estabelecem essa relação, a ausência de evidências empíricas a corroborá-la e, tendo por base fundamentos de inspiração (pós) Keynesiana acerca do comportamento do mercado financeiro, esboçar uma abordagem alternativa.

Palavras-chave: Integração financeira internacional; Instabilidade macroeconômica; Crescimento econômico.

\begin{abstract}
International financial integration and economic growth: a critique to the conventional approach

The arguments in favor of developing an institutional framework for the liberalization of capital flows among countries are based on theoretical models that underline a set of channels through which the International Financial Integration would lead to a better resource allocation, stimulating long run economic growth. The aim of this work is to make a critical evaluation of those models, emphasizing their main fragilities and the lack of empirical evidence to support them. In addition, this paper outline an alternative approach, a Post-Keynesian point of view.
\end{abstract}

Key words: International financial integration; Macroeconomic instability; Economic growth. JEL: F33, F36, F43.

\section{Introdução}

O compromisso com a manutenção de controles sobre os fluxos de capitais era um dos principais aspectos do conjunto de arranjos institucionais acordados em Bretton Woods. Todavia, nas últimas três décadas do século passado, de modo geral, as economias nacionais moveram-se em direção a uma maior permissividade quanto à liberdade para a circulação de capitais entre as fronteiras nacionais.

(1) Trabalho recebido em novembro de 2006 e aprovado em março de 2007.

(2) Professor do Instituto de Economia da Universidade Federal de Uberlândia (IE-UFU) e Doutorando em Economia pelo Instituto de Economia da Universidade Estadual de Campinas (IE-Unicamp). 
O processo de integração financeira foi mais acentuado nos países desenvolvidos, tendo seu início no começo dos anos 1970 no bojo da desarticulação do arcabouço institucional erigido em Bretton Woods, se intensificando a partir dos anos 1980 e, principalmente, ao longo da década de 1990. Nos países em desenvolvimento esse movimento adquire forma mais acabada a partir do final da década de 1980 e se intensifica ao longo da década de 1990.

Do ponto de vista teórico, a defesa de um desenho institucional para as economias domésticas que permita a livre circulação de capitais entre as fronteiras é fundamentada por modelos teóricos que sublinham um conjunto de canais por meio dos quais a Integração Financeira Internacional levaria à melhoria na eficiência da alocação de recursos, estimulando o crescimento econômico de longo prazo dos países.

Em recente estudo realizado no âmbito do Fundo Monetário Internacional, Prasad et al. (2003, p. 37) questionam "why is it so difficult to find a strong and robust effect of financial integration on economic growth for developing countries, when the theoretical basis for this result is apparently so strong?". A resposta dos autores é sumarizada na seguinte frase: "Of course, the lack of a strong and robust effect of financial integration on economic growth does not necessarily imply that theories that make this connection are wrong. Financial Integration without a proper set of preconditions might lead to few growth benefits [...]" (Prasad et al., 2003, p. 37).O objetivo do trabalho é realizar uma crítica a essa abordagem, mostrando as fragilidades das teorias que estabelecem essa relação, a ausência de evidências empíricas a corroborá-la e, tendo por base fundamentos de inspiração (pós) Keynesiana acerca do comportamento do mercado financeiro, esboçar uma visão alternativa.

O texto está organizado em cinco seções além desta introdução; i) nas seções 1 e 2 é apresentada a abordagem convencional acerca da relação entre Integração Financeira Internacional e crescimento econômico; ii) na seção 3 é apresentada e discutida a literatura empírica acerca da relação entre Integração Financeira Internacional e crescimento econômico; iii) na seção 4 é apresentada a abordagem de Stiglitz acerca da relação entre Integração Financeira Internacional e crescimento econômico; iv) na seção 5, faz-se uma crítica à abordagem convencional a parir de fundamentos teóricos de inspiração (pós) Keynesiana e tenta-se esboçar uma abordagem alternativa; vi) e, por fim alinham-se algumas considerações finais.

\section{Benefícios potenciais da integração financeira internacional}

A literatura convencional identifica um conjunto de canais por meio dos quais a Integração Financeira Internacional levaria à melhoria na eficiência da 
alocação de recursos, estimulando o crescimento econômico de longo prazo dos países. No que segue, procura-se deixar claro quais seriam esses canais.

\subsection{Aumento da poupança doméstica}

Do ponto de vista da economia internacional, a Integração Financeira Internacional possibilitaria ativar o mecanismo de intermediação global de recursos entre poupadores e investidores. Como argumenta Fisher (1998, p. 2),

Put abstractly, free capital movements facilitate an efficient global allocation of savings and help channel resources into their most productive uses, thus increasing economic growth e welfare [...] From the viewpoint of the international economy, open capital accounts support the multilateral trading system by broadening the channels through which countries can finance trade and investment and attain higher levels of income.

Esse aumento da eficiência na alocação global de recursos deriva do teorema de Hecksher-Ohlin, segundo o qual a taxa de retorno dos fatores de produção em cada país depende da disponibilidade relativa dos fatores. Desse modo, nos países com abundância de capital, o rendimento real do investimento marginal seria em geral mais baixo do que em países com escassez de capital. O livre fluxo de capitais, ao permitir a busca de retornos marginais maiores, reduziria a escassez relativa de capital nos países com escassez relativa desse fator, de modo que tenderia a haver uma homogeneização dos preços dos ativos e das taxas de juros (Prates, 1997).

Do ponto de vista das economias domésticas, a mobilização da poupança externa é o papel clássico atribuído aos fluxos de capitais em um país em desenvolvimento. $\mathrm{O}$ argumento é que os influxos líquidos de poupança externa podem complementar a poupança interna, elevar o investimento e acelerar o crescimento. Ou seja, "The most straightforward channel through which foreign finance can lift up the growth rate of a country is to supplement domestic saving and correspondingly to raise capital accumulation" (Cohen, 1994, p. 222).

A idéia é que em países em desenvolvimento, além da escassez de capital, os baixos níveis de renda e de poupança constituiriam restrições ao crescimento. Logo, a poupança externa, na forma de entrada líquida de capitais, poderia complementar a poupança interna e, assim, elevar as taxas de investimento e crescimento econômico. Em seguida, o aumento da renda possibilitaria a elevação da poupança e investimento doméstico, criando um círculo virtuoso no qual há uma expansão econômica sustentada. Esse argumento é exposto de forma clara em Devlin et al. (1994, p. 414):

De fato, influxos líquidos de poupanças externas podem suplementar a poupança interna, elevar o investimento e acelerar o crescimento. Em sequiência, a expansão da renda agregada pode elevar ainda mais a poupança e o investimento interno, criando assim um círculo virtuoso no qual há uma expansão econômica sustentada $[\ldots]$ 


\subsection{Redução nos custos do capital}

A liberalização do mercado de capital teria como resultado uma melhora na alocação do risco. Primeiro, o aumento de oportunidades para compartilhar risco entre investidores externos e domésticos pode ajudar a diversificar risco. Essa possibilidade para diversificação de riscos por sua vez estimula firmas a empreenderem mais projetos de investimento, e desse modo aumenta o crescimento econômico. Segundo, quando os fluxos de capitais aumentam, o mercado de capital torna-se mais líquido, o que pode reduzir o prêmio de risco das ações e títulos, e desse modo diminuir o custo de levantar capital para investimentos (Prasad et al., 2003).

Ainda tendo em vista a possibilidade de diversificação de portfólio resultante da Integração Financeira Internacional, McLean e Shrestha (2002) argumentam que a oportunidade para diversificar risco pode aumentar o crescimento econômico por induzir uma mudança em direção a investimentos em projetos com altas taxas de retorno esperado. Por sua vez, altas taxas de retorno podem estimular o crescimento econômico por estimular altas taxas de poupança e investimento. Argumento exposto por Eichengreen et al. $(1998$, p. 12) vai na mesma direção:

Capital mobility can thereby enable investors to achieve higher risk-adjusted rates of return. Income levels in recipient countries should also rise as a result of the capital inflows. Higher rates of return can encourage saving and investment that deliver faster rates of economic growth.

\subsection{Transferência de tecnologia}

Prasad et al. (2003) argumentam que economias financeiramente integradas tendem a atrair grande parte dos fluxos de capitais na forma de Investimento Externo Direto, que tem o potencial de gerar spillovers tecnológicos e serve como canal para a transmissão de melhores práticas administrativas. Esses spillovers podem aumentar a produtividade agregada e, por sua vez, estimular o crescimento econômico. A idéia é que, além de representar um acréscimo à poupança doméstica, o Investimento Externo Direto pode estimular o crescimento econômico por meio da transferência de tecnologia, técnicas administrativas e uma melhor qualificação da força de trabalho, argumento que também é exposto em Agénor (2003, p. 7):

FDI may facilitate the transfer or diffusion of managerial and technological knowhow - particularly in the form of new varieties of capital inputs - and improve the skills composition of the labor force as a result of "learning by doing" effects, investment in formal education, and on-the-job training. In addition, although the increased degree of competition in the product and factor markets induced by FDI may tend to reduce profits of local firms, spillover effects through linkages to supplier industries may reduce input costs, raise profits, and stimulate domestic investment. 


\subsection{Desenvolvimento do sistema financeiro doméstico}

A Integração Financeira Internacional incrementaria a eficiência do sistema financeiro doméstico devido à possibilidade de especialização na oferta de serviços financeiros e à maior concorrência entre instituições residentes e não residentes. Além disso, a integração com o mercado financeiro internacional reduziria a margem de intermediação e abriria acesso ao mercado externo e, assim, a créditos com menores custos a empresas dos países em desenvolvimento (Prates, 1997). Como argumentam Klein e Olivei (1999, p. 1):

Another possible reason for favoring open capital markets is that foreign borrowing and lending may contribute to the development of a country's financial system. A well-functioning financial system provides a set of markets for borrowing and lending, which mitigates problems of asymmetric information and transaction costs, thereby mobilizing savings, efficiently allocating resources, facilitating risk management, exerting corporate control, and easing the trade of goods and services. [...] Thus, opening capital markets, by promoting financial depth, also promotes overall economic growth.

Ou seja, a Integração Financeira Internacional pode contribuir para o crescimento econômico por meio dos seus efeitos no sistema financeiro doméstico, argumento que também é exposto por Agénor (2003, p. 11):

An increasingly common argument in favor of financial openness is that it may increase the depth and breadth of domestic financial markets and lead to an increase in the degree of efficiency of the financial intermediation process, by lowering costs and "excessive" profits associated with monopolistic or cartelized markets. In turn, improved efficiency may lead to lower markup rates in banking, a lower cost of investment, and higher growth rates.

\subsection{Disciplina macroeconômica}

Tem sido argumentado que, por aumentar a recompensa para a prática de 'boas políticas econômicas' e as penalidades para a prática de 'más políticas econômicas', a livre mobilidade de capitais entre as fronteiras pode induzir os países a seguirem políticas macroeconômicas mais disciplinadas e, desse modo, reduzir a freqüência de erros na condução da política econômica. Na medida em que a prática de políticas econômicas disciplinadas se traduza em grande estabilidade macroeconômica e financeira, o resultado pode ser altas taxas de crescimento econômico devido a uma alocação mais eficiente de recursos (Agénor, 2003). Prasad et al. (2003, p. 26), da mesma maneira, chamam a atenção para os benefícios da disciplina macroeconômica imposta pela Integração Financeira Internacional:

[...] the disciplining role of financial integration could change the dynamics of domestic investment in an economy to the extent that it leads to a reallocation of capital towards more productive activities in response to changes in macroeconomic policies. National governments are occasionally tempted to 
institute predatory tax policies on physical capital. The prospect of such policies tends to discourage investment and reduce growth. Financial opening can be selfsustaining and constrains the government from engaging in such predatory policies in the future since the negative consequences of such actions are far more severe under financial integration.

\section{Assimetria de informação e distorções domésticas}

Eatwell (1996) argumenta que está implícito nos argumentos em favor da Integração Financeira Internacional a primeira parte do Teorema Fundamental da Economia do Bem-Estar combinado com a Hipótese de Mercados Eficientes.

$\mathrm{O}$ argumento de que mercados competitivos são eficientes e, portanto, que controles de capitais são ineficientes, baseia-se no Teorema Fundamental, sendo que, um corolário do mesmo argumento propõe que, na ausência de distorções, economias tipicamente operam ou na plena capacidade ou próximo dela (Eatwell, 1996, p. 7).

A Hipótese de Mercados Eficientes retrata o mercado financeiro como eficiente aglutinador e transmissor de informações. Quando essa informação inclui o conhecimento do 'verdadeiro' comportamento da economia (os fundamentos), então os ativos financeiros embutem o valor verdadeiro de suas contrapartes reais, criando um ambiente no qual agentes racionais comercializando esses ativos podem tomar decisões Pareto-eficientes (Eatwell, 1996, p. 8).

Parte da literatura convencional chama a atenção para a possibilidade de que os benefícios potenciais da Integração Financeira Internacional não se concretizem como resultado de distorções. McLean e Shrestha (2002, p. 8) argumentam que na presença de distorções, fluxos de capitais "would not enhance growth, and can in fact impede growth by making countries more vulnerable to financial crises". A argumentação de Bailliu (2000, p. 4), a respeito de fluxos de capitais na presença de distorções, vai na mesma direção,

Capital flows occurring in this context are not likely to be growth-promoting. Indeed, even though capital inflows motivated by distortions might lead to an increase in the investment rate, this will not contribute to higher growth unless the investment is productive. In terms of spillovers, this kid of speculative investment is not likely to generate any positive externalities, either in the financial or nonfinancial sectors of the economy.

Há, na literatura convencional, pelo menos dois conjuntos de argumentos que procuram justificar, teoricamente, a possibilidade de não-concretização dos potenciais benefícios da Integração Financeira Internacional: i) a presença de assimetria de informação nos contratos financeiros; e ii) existência de distorções no funcionamento das economias domésticas. 


\subsection{Assimetria de informação}

A informação é assimétrica quando uma parte de uma transação ou relação econômica tem menos informação sobre ela do que a outra parte. Sob o pressuposto de existência de assimetria de informação, ineficiências podem surgir como resultado de três problemas: Seleção adversa, Risco moral e Comportamento de manada. Ou seja, como observam Eichengreen et al. (1998, p. 12): "The classic case for unfettered capital markets is predicated on the assumption that they deliver an efficient allocation of resources. [...] Under the alternative assumption that information is asymmetric, inefficiencies can arise".

\subsubsection{Seleção adversa}

Seleção adversa é um problema de assimetria de informação que ocorre antes da transação acontecer devido ao fato de os credores terem incompleto conhecimento acerca da qualidade dos tomadores de empréstimos, sendo que os tomadores de empréstimos que têm um alto risco de crédito são os que buscam mais ativamente tomar empréstimos.

A presença de assimetria de informação torna os credores menos hábeis para avaliarem a qualidade das operações de crédito. Nestas condições, os emprestadores estarão dispostos a pagar um preço por um título que reflete a qualidade média dos títulos emitidos pelas firmas, de modo que esse preço provavelmente será menor que o valor de mercado dos títulos emitidos por firmas de baixo risco de crédito, mas acima do valor de mercado dos títulos emitidos por firmas de alto risco de crédito. Pelo fato de administradores e proprietários de firmas com baixo risco de crédito perceberem que os títulos deles estão subvalorizados, eles não irão querer se financiar via mercado. Por outro lado, as firmas que desejarão vender títulos no mercado são aquelas de baixa qualidade de crédito, porque sabem que os preços de seus títulos são maiores que seus valores de mercado. Como resultado da pequena quantidade de títulos emitidos por firmas com baixo risco de crédito, muitos projetos com um valor presente líquido positivo não serão empreendidos, embora outros, cujo valor presente líquido é menor que o custo de oportunidade dos fundos alocados, sejam. Sob essas circunstâncias, um mercado de capitais liberalizado não gera uma alocação eficiente de recursos (Eichengreen et al., 1998; Mishkin, 1996).

\subsubsection{Risco moral}

Risco moral ocorre após a transação ter acontecido porque o emprestador está sujeito ao risco (hazard) decorrente do fato de que o tomador de empréstimo tem incentivo para engajar em atividades que são indesejáveis (immoral) do ponto 
de vista do emprestador, isto é, atividades que tornam o empréstimo mais arriscado. Os tomadores de empréstimo têm incentivos para investir em projetos com altos riscos nos quais eles 'se dão bem' se o projeto tem êxito, mas os emprestadores arcam com a maioria das perdas se o projeto fracassa. Portanto, tomadores de empréstimos tentarão alterar seus projetos de maneira que os riscos aumentem após a transação ter ocorrido, sendo que a assimetria de informação facilitará os esforços deles para tanto. Sob essas circunstâncias, muitos projetos em execução serão excessivamente arriscados. Os emprestadores, como resultado, ficarão relutantes em fazer empréstimos e o nível de intermediação e investimento será subótimo (Eichengreen et al., 1998; Mishkin, 1996).

\subsubsection{Comportamento de manada}

Em mercados financeiros caracterizados por incompleta informação, emprestadores podem ser propensos a engajar em Comportamento de manada, por meio do qual eles tendem a seguir a liderança daqueles que eles acreditam estarem melhores informados. Tal comportamento dá origem a repentinos movimentos de mercado e volatilidade.

Como argumentado em Agénor (2003), Comportamento de manada pode ser considerado racional na presença de três fatores: i) o fato de que o payoff para um agente (investidor) adotando uma ação específica pode ser positivamente relacionado com o número de outros agentes adotando a mesma ação; ii) o fato de que administradores de portfólio podem ter incentivos para 'hide in the herd', de modo a não serem avaliados facilmente; iii) o fato de pequenos investidores que estão começando a investir em um país ignorarem suas próprias informações e seguir o comportamento de investidores maiores e melhores estabelecidos.

\subsection{Distorções domésticas}

Mesmo quando a informação é completa, a Integração Financeira Internacional pode não resultar em estímulo ao crescimento econômico dos países (senão o contrário), como resultado da presença de distorções no funcionamento das economias domésticas. As distorções dizem respeito a práticas de políticas comerciais protecionistas e baixo nível de desenvolvimento do sistema financeiro doméstico.

A mobilidade internacional de capitais na presença de significativas distorções em relação ao comércio resultará em uma má alocação do capital mundial e, certamente, até mesmo uma piora do bem-estar do país importador de capital. Considerando um país relativamente abundante em trabalho que protege suas indústrias intensivas em capital, devido ao fato da proteção a essas indústrias 
estimular a taxa de retorno para o capital investido no país, os capitais fluirão em direção a esse país, levando os setores intensivos em capital a expandirem e os setores intensivos em trabalho a contraírem. Isso resulta em diminuição dos níveis de renda e bem-estar, porque a má alocação de recursos entre setores intensivos em trabalho e setores intensivos em capital é exacerbada por conta da Integração Financeira Internacional, dificultando a operação do mecanismo das vantagens comparativas. Nesse sentido, Cooper $(1998$, p. 13) conclui que "free movement of capital is likely to become allocationally efficient only after trade barriers have come down substantially, particularly barriers on capital-intensive activities in labor-rich countries". Essa idéia também fica bastante clara na argumentação de Edison; Levine; Ricci e Sloek (2002, p. 1):

[...] there are innumerable constellations of distortions for which liberalization of international capital controls will hurt resource allocation and growth. For example, in the presence of trade distortions, capital account liberalization may induce capital inflows to sectors in which the country has a comparative disadvantage.

A má alocação do capital também pode ser resultado de distorções preexistentes no sistema financeiro doméstico. $\mathrm{O}$ argumento é que em países com fraco sistema bancário e pobre supervisão e regulação, a intermediação direta ou indireta de grande montante de fundos pelo sistema bancário pode exacerbar problemas de Risco Moral associados com garantias explícitas ou implícitas de depósitos (Agénor, 2003; Bailliu, 2000; Soto, 2000).

Tendo isso em vista, Eichengreen et al. (1998, p. 14) afirmam que “[...] the theoretical presumption in favor of the liberalization of domestic and international financial markets is weakened by the presence of asymmetric information and domestic distortions", sendo que a possibilidade de potenciais benefícios dependeria da natureza das distorções, extensão da assimetria de informação, e a severidade da Seleção adversa, Risco moral e ineficiências de mercado resultantes. No entanto, o autor argumenta que esses problemas podem ser corrigidos ou diminuídos significativamente por meio de políticas públicas.

Para as distorções domésticas que são fundamentalmente resultantes de políticas governamentais equivocadas, a solução proposta é a correção dessas políticas. Se influxos de capitais ameaçam reduzir o bem-estar porque fluem em direção a indústrias domésticas fortemente protegidas onde o verdadeiro retorno para o capital é menor que os custos dos empréstimos externos, então a política correta sugerida é eliminar ou reduzir a proteção para corrigir as distorções.

Com relação aos problemas de assimetria de informação, Eichengreen et al. (1998) sugerem que podem ser amenizadas por meio de políticas públicas, notadamente: i) políticas que estimulem a aderência aos padrões mundiais de contabilidade e auditoria; ii) que facilite o cumprimento de regras sadias de 
governança corporativa; e iii) que proteja investidores e emprestadores contra fraudes. A conclusão dos autores é que:

Capital account liberalization and financial liberalization more generally, is essentially inevitable for all countries wishing to take advantage of the substantial benefits of broad participation in the open world economic system in this modern age of technology and communications. Financial liberalization also has its dangers, as liberalized systems generally afford opportunities for individuals, enterprises, and financial institutions to undertake greater and sometimes imprudent risks, thereby raising the potential for systemic disturbances. There is no way to completely suppress these dangers, other than through draconian financial repression, which creates worse problems. The dangers, however, can be limited quite considerably through a combination of sound macroeconomic policies to contain aggregate financial imbalances and ameliorate the effects of financial disturbances and sound prudential policies to ensure proper private incentives for risk management. These must be back up by adequate supervision and regulation, especially of the financial sector. With these important safeguards, capital account liberalization and broader financial liberalization are not only inevitable, but will clearly be beneficial (Eichengreen et al., 1998, p. 1).

Alguns autores chamam a atenção para a importância do nível de desenvolvimento institucional dos países na relação entre Integração Financeira Internacional e crescimento econômico. A idéia é que a Integração Financeira Internacional promoverá crescimento econômico somente em países institucionalmente desenvolvidos. A esse respeito:

[...] IFI in countries with weak institutions and policies - e.g., weak financial and legal systems - may actually induce a capital outflow from capital-scarce countries to capital-abundant countries with better institutions. Thus, some theories predict that international financial integration will promote growth only in countries with sound institutions and good policies (Edison; Levene; Ricci; Sloek, 2002, p. 1)

Ao considerar as implicações da existência de assimetria de informação no mercado financeiro e distorções no funcionamento das economias domésticas para a relação entre Integração Financeira Internacional e crescimento econômico, emerge dessa discussão que seria necessário um conjunto de precondições a ser preenchido pelas economias nacionais para que os potenciais benefícios da Integração Financeira Internacional se concretizassem: i) certo nível de desenvolvimento do sistema financeiro doméstico; ii) certo grau de abertura ao comércio internacional; e iii) prática de políticas econômicas consideradas 'sadias' pela literatura convencional. Ou seja, admitido que a presença de assimetria de informação e a existência de distorções no funcionamento das economias domésticas enfraquecem os pressupostos teóricos a favor da Integração Financeira Internacional, os autores argumentam que seria necessária a existência de um conjunto de instituições adequadamente desenvolvidas para que a Integração Financeira Internacional estimulasse o crescimento econômico de longo prazo dos países. 


\section{Apresentação e apreciação crítica da literatura empírica}

Desde o final da década de 1990, é crescente o esforço de pesquisa com o objetivo de verificar empiricamente a existência de benefícios advindos da Integração Financeira Internacional. No geral, os trabalhos estimam equações de crescimento incluindo entre as variáveis explanatórias algum indicador como proxy para o grau de Integração Financeira Internacional dos países, que varia desde proxies para existência de restrições governamentais, até a utilização dos próprios fluxos de capitais como proxy (variáveis de resultado). No que segue, são apresentados brevemente alguns resultados de estudos empíricos e, em seguida, feita uma apreciação crítica desta literatura.

\subsection{A literatura empírica}

O estudo empírico pioneiro foi elaborado por Quinn (1997), quem construiu um indicador de Integração Financeira Internacional baseado em diversos números do Annual Report on Exchange Arrangements and Exchange Restrictions divulgado pelo Fundo Monetário Internacional. O autor utilizou esse índice nas equações de crescimento estimadas para uma amostra de países desenvolvidos e em desenvolvimento no período 1960-1989. Os resultados econométricos, de acordo com Quinn (1997), proporcionavam a primeira demonstração sistemática de que a Integração Financeira Internacional contribui para o crescimento econômico de longo prazo dos países.

Rodrik (1998), utilizando como indicador de Integração Financeira Internacional uma variável binária disponibilizada pelo Fundo Monetário Internacional até 1995, que informa a ausência ou presença de restrições, estimou equações de crescimento utilizando dados para países desenvolvidos e em desenvolvimento no período 1975-1989. A conclusão do trabalho é que "the data provide no evidence that countries without capital controls have grown faster, invested more, or experienced lower inflation" (Rodrik, 1998, p. 61). Também não foram encontradas evidências de que a Integração Financeira Internacional teria efeitos positivos sobre o crescimento econômico de países com alto grau de desenvolvimento institucional:

The logic of this view suggests that capital-account convertibility should have beneficial effects in countries with strong institutions. I find no evidence for this on the data: Interacting capital-account liberalization with indices of the quality of public institutions yields insignificant (and often "wrong" - signed) coefficients (Rodrik, 1998, p. 63).

Edison et al. (2002), em estudo elaborado no âmbito do Fundo Monetário Internacional, estimaram equações de crescimento para uma amostra contendo países desenvolvidos e em desenvolvimento no período 1980-1995. São utilizados 
como proxies para o grau de Integração Financeira Internacional dos países tanto a variável fornecida pelo Fundo Monetário Internacional quanto fluxos brutos de capitais privados. A conclusão dos autores é que os resultados econométricos não corroboram a hipótese de que a Integração Financeira Internacional estimula o crescimento econômico, mesmo para países com alto grau de desenvolvimento institucional, alto grau de desenvolvimento financeiro e que praticam políticas econômicas consideradas sadias pela literatura convencional.

Também em estudo elaborado no âmbito do Fundo Monetário Internacional, Edison et al. (2002) estimaram equações de crescimento com dados para uma amostra contendo países desenvolvidos e em desenvolvimento no período 1976-1995, utilizando como medidas de Integração Financeira Internacional a variável binária fornecida pelo Fundo Monetário Internacional e o índice elaborado por Quinn (1997). Os resultados do trabalho sugerem que os efeitos positivos da Integração Financeira Internacional são maiores e mais significativos entre os países em desenvolvimento do que nos países desenvolvidos e entre aqueles nos países do Leste da Ásia.

Com o objetivo de testar se Influxos de Capitais Totais, Investimento Externo Direto, Investimento de Portfólio e Créditos Bancários exercem um efeito positivo sobre o crescimento de longo prazo dos países, McLean e Shrestha (2002) estimam equações de crescimento utilizando dados para uma amostra contendo países desenvolvidos e em desenvolvimento. A conclusão do trabalho é que "We find that foreign direct investment and portfolio inflows enhance growth. By contrast, bank inflows appear to have a negative effect on growth [...]" (McLean; Shrestha, 2002, p. 18).

Da mesma maneira, com o objetivo de testar o efeito de fluxos de Investimento Externo Direto e Investimento de Portfólio sobre o crescimento econômico de longo prazo dos países, Carkovic e Levine (2002) estimam equações de crescimento para uma amostra contendo países desenvolvidos e em desenvolvimento no período 1965-1995. A conclusão do trabalho é que Investimento Externo Direto e Investimento de Portfólio não exercem efeito positivo sobre o crescimento econômico de longo prazo, nem mesmo em países com alto grau de desenvolvimento econômico, com alto grau de desenvolvimento do sistema financeiro doméstico, com grande estoque de capital humano e alto grau de abertura ao comércio internacional. Ou seja,

This paper's findings are robust to (a) econometric specification that allow FDI to influence growth differently depending on national income, school attainment, domestic financial development, and openness to international trade, (b) alternative estimation procedures, (c) different conditioning information set and samples, (d) the use of portfolio inflows instead of FDI, and (e) the use of alternative databases on FDI. The data produce consistent results: there is not a robust, causal link running from FDI to economic growth (Carkovic; Levine, 2002, p. 3). 
Essa pequena síntese da literatura empírica sugere, no mínimo, que não existe consenso de que a Integração Financeira Internacional tenha efeitos positivos sobre o crescimento econômico de longo prazo dos países. Com o objetivo de realizar uma apreciação crítica dessa literatura, o próximo passo é procurar na literatura convencional argumentos que sejam arrolados para explicar essa ausência de evidências robustas.

\subsection{Apreciação crítica da literatura empírica}

Segundo Rogoff (2002), economista-chefe do Fundo Monetário Internacional na ocasião, a intenção desta instituição em promover a convertibilidade da conta de capital dos seus países membros durante a década de 1990, que teve seu apogeu na reunião anual de 1997 realizada em Hong Kong, revelava uma preferência à priori, mais do que o resultado fundamentado do trabalho de pesquisa da instituição. Stiglitz (2000, p. 1076) também chama a atenção para a falta de fundamentação teórica, empírica (econométrica) e histórica na perspectiva liberalizante do Fundo Monetário Internacional:

The case for capital market liberalization was found wanting, especially striking given the zeal with which the International Monetary Fund (IMF) had requested an extension of its mandate to include capital market liberalization a short two years earlier at the Annual Meetings in Hong Kong. It should have been clear then, and it is certainly clear now, that the position was maintained either as a matter of ideology or of special interests, and not on the basis of careful analysis of theory, historical experience or a wealth of econometric studies.

O atual estágio dessa literatura, ao revelar a inexistência de consenso quanto a uma relação causal robusta e positiva da Integração Financeira Internacional para o crescimento econômico, levou ao seguinte questionamento, apresentado na introdução a este trabalho: "Why is it so difficult to find a strong and robust effect of financial integration on economic growth for developing countries, when the theoretical basis for this result is apparently so strong?" (Prasad et al., 2003, p. 31). A explicação dos autores para essa ausência de evidências empíricas também apresentada na introdução a este trabalho, reflete sobremaneira a posição da literatura convencional acerca do assunto e é sumarizada na seguinte frase: "Of course, the lack of a strong and robust effect of financial integration on economic growth does not necessarily imply that theories that make this connection are wrong. Financial Integration without a proper set of preconditions might lead to few growth benefits [...]" (Prasad et al., 2003, p. 37). Essa é, não por acaso, a atual posição do Fundo Monetário Internacional relativamente à Integração Financeira Internacional, como é bem ressaltado por Carvalho (2004, p. 1):

Na verdade, mesmo após o FMI ter reconhecido (ao final da década de 1990) que a liberalização dos movimentos de capitais foi precipitada em muitos casos, 
defendendo que a remoção dos controles restantes seja feita após o seqüenciamento correto de reformas domésticas prévias, não é possível encontrar exemplos de países que tenham sido autorizados pela instituição a reinstalar controles para que aquelas reformas possam ser implementadas. O que o FMI defende hoje pode ser chamado de liberalização cautelosa, mas o viés em favor da liberalização permanece.

Antes de expor e discutir os argumentos arrolados por Prasad et al. (2003) com vistas a explicarem os resultados encontrados na literatura empírica, é necessário ressaltar que a pergunta dos autores assume implicitamente que existe consenso relativamente à existência de uma relação causal positiva e robusta entre Integração Financeira Internacional e crescimento econômico de longo prazo para os países desenvolvidos, o que não é verdade. Trabalho elaborado no âmbito do próprio Fundo Monetário Internacional, examinado na subseção anterior, não encontra evidências de que a Integração Financeira Internacional estimula o crescimento econômico de longo prazo dos países desenvolvidos. Para deixar claro, a conclusão do trabalho é a seguinte: "The results of this paper, however, suggest that open capital accounts and liberalized stock markets have a significant effect on growth among East Asian countries, but there is less consistent evidence of these effects elsewhere, even among industrial countries" (Edison et al., 2002, p. 35). Além disso, os autores argumentam que os resultados encontrados para os países do Leste da Ásia podem está refletindo o fato de que o indicador de Integração Financeira Internacional utilizado seja uma proxy para outras características das economias.

No que segue, são apresentados e discutidos os argumentos de Prasad et al. (2003), ou seja, as precondições identificadas pelos autores, sem as quais a Integração Financeira Internacional poderia resultar em poucos benefícios em termos de crescimento econômico:

i) o primeiro argumento apresentado diz respeito ao fato de que a Integração Financeira Internacional na presença de distorções em relação ao comércio internacional pode não resultar em benefícios em termos de crescimento econômico. Ou seja, os benefícios dependeriam do grau de abertura das economias ao comércio internacional. Essa argumentação é apresentada de forma bastante clara na seguinte passagem:

It is useful to note that there may be a complementary relationship between trade and financial openness. For example, if a country has severe trade barriers protecting some inefficient domestic industries, then capital inflows may end up being directed to those industries, thereby exacerbating the existing misallocation of resources. Thus, there is a concrete channel through which financial openness without trade openness could lower a country's level of efficiency (Prasad et al., 2003, p. 37). 
Esse argumento foi testado e não corroborado por trabalhos empíricos. Carkovic e Levine (2002), em trabalho examinado na subseção anterior, testam a hipótese de que os efeitos dos influxos de Investimento Externo Direto e Investimento de Portfólio sobre o crescimento econômico dependeriam do grau de abertura das economias domésticas ao comércio internacional e não encontram evidências empíricas que corroborem essa hipótese.

ii) o segundo argumento diz respeito ao fato de que os benefícios da Integração Financeira Internacional em termos de estímulo ao crescimento econômico dependeriam do grau de desenvolvimento do sistema financeiro doméstico. De acordo com esse argumento, a Integração Financeira Internacional na presença de um setor financeiro doméstico pouco desenvolvido pode não gerar benéficos porque os influxos de capitais exacerbariam as ineficiências alocativas existentes nas economias. Ou seja:

In the presence of weakly regulated banking systems and other distortion in domestic capital markets, inflows of foreign could exacerbate the existing inefficiencies in these economies. For example, if domestic financial institutions tend to channel capital to firms with excessive risks or weak fundamentals, financial integration could simply lead to an intensification of such flows (Prasad et al., 2003, p. 50).

Esse argumento, de que os efeitos da Integração Financeira Internacional dependeriam do grau de desenvolvimento do sistema financeiro doméstico, também já foi testado por trabalhos empíricos. A esse respeito, Edison et al. (2002), em trabalho examinado na subseção anterior, argumentam que "the results do not reject the null hypothesis that IFI is unrelated to economic growth even when allowing this relationship to vary with financial development".

iii) O terceiro argumento é de que os benefícios advindos dos influxos de Investimento Externo Direto dependeriam do nível de capital humano nos países receptores. A idéia é que somente países com nível de capital humano suficientemente alto podem explorar os spillovers tecnológicos associados com o Investimento Externo Direto. A esse respeito:

[...] there is some evidence that the effect of foreign direct investment on growth depends on the level of human capital in a developing country. For countries with relatively low human capital, there is at best a small positive effect that can be detected in the data. On the other hand, for countries whose human capital has exceeded a certain threshold, there is some evidence that FDI promotes economic growth (Prasad et al., 2003, p. 50).

O estudo elaborado por Carkovic e Levine (2002), apresentado na subseção anterior, testa um conjunto amplo de hipóteses, inclusive esta arrolada por Prasad et al. (2003). Os autores não encontram evidências nem de efeitos positivos do Investimento Externo Direto sobre o crescimento econômico, nem evidências de que esse efeito dependeria do nível de capital humano nas 
economias domésticas ou de um conjunto ainda maior de precondições, como nível de abertura ao comércio internacional, nível de desenvolvimento do sistema financeiro doméstico e nível de desenvolvimento econômico.

iv) $\mathrm{O}$ quarto argumento diz respeito ao fato de que a relação entre Integração Financeira Internacional e crescimento econômico dependeria do grau de desenvolvimento institucional das economias, estabilidade macroeconômica e práticas de políticas macroeconômicas consideradas 'sadias' pela literatura convencional. Isso fica claro nas seguintes passagens:

[...] financial globalization, in combination with good macroeconomic policies and good domestic governance, appears to be conducive to growth (Prasad et al., 2003, p. 10).

More generally, one might think of a country's absorptive capacity in terms of human capital, depth of domestic financial market, quality of governance and macroeconomic policies. There is some preliminary evidence that foreign capital flows do not seem to generate positive productivity spillovers to domestic firms for countries with a relatively low absorptive capacity, but positive spillovers are more likely to be detected for countries with a relatively high level of absorptive capacity. This evidence is consistent with the view that countries need to build up a certain amount of absorptive capacity in order to effectively take advantage of financial Globalization (Prasad et al., 2003, p. 50).

Todas essas hipóteses foram testadas na literatura empírica. Rodrik (1998), em trabalho examinado na subseção anterior, testa a hipótese de que a Integração Financeira Internacional tem efeitos positivos sobre o crescimento econômico em países institucionalmente desenvolvidos e não encontra evidências empíricas robustas. Estudo realizado no âmbito do Fundo Monetário Internacional e apresentado na subseção anterior também não encontra evidências empíricas que corroborem os argumentos de Prasad e et al. (2003). Ou seja, os autores concluem que: "The data do not support the view that international financial integration per se accelerates economic growth even when allowing this relationship to vary with economic, financial, institutional, and macroeconomic characteristics" (Edison et al., 2002, p. 24).

Como deve ter ficado claro, as hipóteses levantadas por Prasad et al. (2003) para explicarem a dificuldade de encontrar, nos trabalhos empíricos, consenso quanto a uma relação causal robusta e positiva entre Integração Financeira Internacional e crescimento econômico, já foram testadas e não corroboradas pela literatura empírica. Quando essa literatura era ainda incipiente, essa ausência de evidências levou Rodrik (1998, p. 64) a afirmar que "The benefits of removing capital controls, however, remain to be demonstrated". O atual estágio de desenvolvimento da literatura empírica, com crescente sofisticação da modelagem econométrica e utilização de vários indicadores como proxy para Integração Financeira Internacional, dá a essa afirmação uma contemporaneidade 
e significado ainda mais importantes. Portanto, são precipitadas e equivocadas afirmações acerca da comprovação dos benefícios da Integração Financeira Internacional:

Among the demonstrated benefits of the liberal capitalist world system are: i) Liberalized financial systems help to promote growth through financial intermediation and efficient allocation of capital for investment; ii) Private capital flows - both direct foreign investment and portfolio flows - have generally helped increase investment and growth in developing economies (Boorman; Allen, 2000, p. 103).

Além da modelagem econométrica, utilizada como instrumental para investigar a existência de benefícios advindos da Integração Financeira Internacional, é possível encontrar, no âmbito da literatura convencional, autores que se valem de argumentos históricos, apresentando-os como evidências empíricas suficientemente robustas para comprovarem os benefícios da Integração Financeira Internacional:

[...] this experience across a wide range of industrial and developing countries is probably the most important and powerful empirical evidence about the bottom-line questions of whether, on balance, financial liberalization generally and capital account liberalization specifically are beneficial. Countries that have successfully liberalized their financial systems domestically and internationally seek to preserve and develop these systems (Eichengreen et al., 1998, p. 15).

Esse argumento ignora as assimetrias de poder existentes entre as nações, a pressão exercida por instituições multilaterais sobre os países, os interesses representados por estas, assim como os interesses representados por aqueles que detêm o poder de tomar decisões econômicas nos países. A esse respeito, vale a pena reproduzir texto de Stiglitz (2003, p. 509), que deixa bastante evidente a insuficiência do argumento:

A second related argument sometimes put forward is that countries that have partaken of globalization almost never reverse course; evidently, whatever the costs, the benefits exceed the costs. This argument is subject to the same criticismcountries are keenly aware of the consequences of reversing course, of the pressure imposed, say, by the IMF and the United States. When Indonesia, hearing of the new thinking at the IMF about capital market liberalization, proposed rethinking the issue there, they were quickly batted down. Given their current position of dependence, they felt they had little choice but to follow the dictates of their new economic masters. But there are two further answers. Some countries have reversed on the issue of capital market liberalization, though in order to avoid the wrath of the IMF and the US Treasury, they have done so with some subtlety, e.g., effectively imposing restrictions through the banking system. Finally, in many countries, even those that are 'nominally' democratic, economic policy making, at least in the short run, is under the control of particular groups, When one says, 'they' chose to keep their, say, their capital markets open, the 'they' is likely to be the finance ministry, and the finance ministry may reflect more the interests and perspectives of the financial community than of society as a whole. 
Fisher (1998, p. 2) também se vale de argumentos históricos, afirmando que, entre os motivos para a Integração Financeira Internacional, "The first is that it is an inevitable step on the path of development, which cannot be avoided and should be embraced. After all, the most advanced economies all have open capital accounts". A respeito da fragilidade desse argumento, Carvalho (2004, p. 10) faz o seguinte comentário:

À parte o curioso determinismo histórico abraçado por Fisher, cabe notar o argumento falacioso, que o autor usa com freqüência, de que a liberalização deve ser adotada porque é o que um país desenvolvido faz. É difícil de se acreditar que um autor como Fisher desconheça que a liberalização financeira se deu, em praticamente todos os países industriais depois que o desenvolvimento tinha sido alcançado e não como instrumento de desenvolvimento. Na verdade, esta é uma informação tão trivial e tão conhecida que é impossível não alimentar dúvidas quanto às intenções do autor ao propor falácia tão grosseira.

\section{A crítica de Stiglitz}

O objetivo da análise de Stiglitz (2000) é, primeiro, apresentar argumentos visando ressaltar as fragilidades teóricas subjacentes à hipótese de que a Integração Financeira Internacional estimularia o crescimento econômico de longo prazo dos países. Segundo, explicar porque a Integração Financeira Internacional desestimularia o investimento e crescimento econômico. No que segue é apresentado o primeiro conjunto de argumentos:

i) o argumento de que a Integração Financeira Internacional permitiria uma alocação mais eficiente dos recursos globais, incrementando a poupança dos países em desenvolvimento, tem como base o pressuposto de que o mercado de capitais e financeiro é igual a mercados para bens e serviços ordinários. A falha apontada nesse argumento é o fato de que Stiglitz considera os mercados de capital e financeiro essencialmente diferentes de mercados ordinários para bens e serviços, por conta da existência de assimetria de informação, ou seja:

The central function of capital and financial markets is information-gathering - in particular, assessing which projects and firms are most likely to yield the highest returns, and monitoring to ensure that the funds are used in the appropriate way. Moreover, markets for information are fundamentally different from 'ordinary' markets. For instance, whenever information is imperfect, markets are essentially never constrained Pareto efficient - in marked contrast to standard results for competitive markets with perfect information (Stiglitz, 2000, p. 1079)

ii) Quanto ao argumento de que a Integração Financeira Internacional promove estabilidade macroeconômica, o autor considera o mais deficiente. A idéia é que a Integração Financeira Internacional está sistematicamente associada a maior instabilidade, pelo fato dos fluxos de capitais serem marcadamente pró- 
cíclicos, exacerbando as flutuações econômicas, quando não são causadores dessas flutuações (Stiglitz, 2000).

No que concerne especificamente à relação entre as diferentes modalidades de fluxos de capitais e crescimento econômico, o autor faz uma distinção bastante clara, considerando consistentes os argumentos que sublinham os benefícios advindos dos influxos de Investimento Externo Direto. Nas suas palavras: "The argument for foreign direct investment, for instance, is compelling. Such investment brings with it not only resources, but technology, access to markets, and (hopefully) valuable training, an improvement in human capital" (Stiglitz, 2000, p. 1076).

Em relação aos influxos de capitais de curto prazo (Investimento de Portfólio), o autor mostra certo ceticismo quanto aos efeitos positivos sobre o crescimento econômico, pelo fato de não acreditar que firmas tomam decisões de investimentos produtivos de longo prazo tendo como base financiamento de curto prazo. Em adição, o autor argumenta que além de não estimular o crescimento econômico, o comportamento dessa modalidade de fluxos de capitais, em uma economia financeiramente integrada, como se verá adiante, desestimularia influxos de Investimento Externo Direto (Stiglitz, 2000).

A segunda parte da argumentação, qual seja, as justificativas para o fato de a Integração Financeira Internacional desestimular o investimento e crescimento econômico, é apresentada na seqüência:

i) o primeiro argumento é de que a Integração Financeira Internacional leva a instabilidade, e essa instabilidade teria efeitos adversos sobre o comportamento do investimento e crescimento econômico. Além da instabilidade, a presença do risco de instabilidade desestimularia investimentos produtivos. O autor esboça pelo menos um canal por meio do qual essa instabilidade pode afetar o crescimento econômico:

I have already referred to the risk imposed by capital market liberalization. The surges of capital, first into the country, and then out, impose tremendous costs, regardless of the exchange rate regime. Under flexible exchange rates, for instance, these surges are likely to lead to large changes in exchange rates, imposing huge risks on every aspect of society. But I am concerned not just with the overall welfare costs, but the adverse effects of growth. Firms facing more highly volatile prices of inputs and outputs will demand a compensating risk premium - with an adverse effect on investment and growth (Stiglitz, 2003, p. 514).

ii) o segundo argumento diz respeito ao fato da instabilidade associada ao comportamento dos fluxos de capitais de curto prazo (Investimento de Portfólio), em um ambiente financeiro integrado, desencorajar influxos de Investimento Externo Direto, que, segundo o autor, é esperado ter efeitos positivos sobre o crescimento econômico (Stiglitz, 2000). 
iii) o terceiro argumento diz respeito ao fato de que a Integração Financeira Internacional pode facilitar a fuga de capitais do país, com efeitos adversos sobre o crescimento econômico. Ou seja, como argumenta Stiglitz (2000, p. 1081), "Opening the capital account can, and has in several countries, facilitated the flow of capital out of the country (rather than, as promised, accelerating the flow of capital into the country), providing another channel for adverse effects".

iv) o quarto argumento diz respeito ao fato de que, em uma economia financeiramente integrada, necessita-se manter grande quantidade de reservas de modo a proteger o país contra a volatilidade do mercado financeiro internacional. A manutenção dessas reservas representa para o país uma transferência de renda líquida para o exterior, já que o custo dos empréstimos excede a remuneração das reservas. Ou seja:

There is an equally compelling argument for why capital market liberalization (at the short end) might be expected to have adverse effects on growth. Countries today are encouraged to maintain adequate reserves, to protect them-selves against volatility in international financial markets (Stiglitz, 2000, p. 1081).

Esses são os argumentos apresentados para explicar tanto as fragilidades teóricas subjacentes aos argumentos que estabelecem uma relação de causalidade positiva da Integração Financeira Internacional para o crescimento econômico, bem como para explicar por que "[...] there are even reasons to expect that capital market liberalization can have negative effects on growth" (Stiglitz, 2000, p. 1080). Desse modo, a argumentação do autor mostra um teor crítico considerável, evidenciando a ausência de consenso teórico no âmbito da literatura convencional acerca do assunto, mesmo considerando que a abordagem de Stiglitz, indo em direção oposta à análise cautelosa de Eichengreen et al. (1998), compartilha com estes o mesmo fundamento teórico, a existência de assimetrias de informação.

Sem embargo, um último ponto, central na argumentação de Stiglitz, necessita ser examinado com mais cuidado - a instabilidade do mercado financeiro, associada ao comportamento dos fluxos de capitais de curto prazo (Investimento de Portfólio). O autor não deixa clara a origem dessa instabilidade, ou seja, não expõe os fundamentos teóricos acerca do comportamento dos agentes econômicos e funcionamento do mercado financeiro.

Dada a importância da análise de Stiglitz para os propósitos deste trabalho, será exposta a discussão feita por Davidson (1998a, 2002) acerca do modelo de comportamento do mercado financeiro subjacente à análise de Stiglitz, de modo a ter clara a origem dessa instabilidade. A esse respeito:

Observed volatile financial market prices are movements - away from fundamental-determined values. This volatility is attributed primarily to the existence of 'noise traders', that is, speculators who mistakenly believe they know how the stock market works and therefore do not have to acquire the correct 
information regarding future outcomes from the fundamentals. Other rational short-term traders feed on these foolish noise traders and thereby ultimately return the market to its fundamental trend value. Stiglitz's explanation of the horrendous speculative volatility that we observe in our world is the 'mistaken belief of all speculators' that they can do better than the market by ignoring fundamentals (Davidson, 2002, p. 183).

Portanto, a explicação para a existência de volatilidade no mercado financeiro diz respeito ao comportamento, nas transações de curto prazo, de agentes que equivocadamente (irracionalmente) ignoram os fundamentos reais de mercado subjacentes aos preços dos ativos financeiros, interferindo com a principal função desse mercado, que é, segundo essa visão, a alocação eficiente de capital. Ou seja:

Old and New Keynesians as well as classical economists all accept the efficient market hypothesis as the applicable description of real world financial markets and therefore they are invoking the ergodic axiom. One logically inevitable conclusion of the efficient market hypothesis is that, as Stiglitz states, the most important social function' of financial markets is to correctly allocate real capital among industries in accordance with reliable information about future rates of return determined by fundamentals (Davidson, 2002, p. 183).

Segundo Davidson (2002) o modelo de Stiglitz assume que: i) existem fundamentos reais de mercado que são imutáveis no sentido de que não podem ser mudados pela ação humana; e ii) esses fundamentos determinam as probabilidades condicionais de resultados futuros (ou um menu de todos possíveis estados do mundo e possíveis contingências) que são refletidos nos preços atuais do mercado financeiro. Desse modo, está implicitamente admitido o axioma da ergodicidade. Esse axioma assegura que a trajetória de longo prazo da economia está imutavelmente pré-programada e embutida nos fundamentos de hoje, de modo que, em um sistema predeterminado como este, o mercado necessariamente alocará capital de forma ótima entre projetos alternativos, contanto que os agentes sejam livres para tomarem decisões de mercado baseadas em informação corrente estatisticamente confiável em relação a taxas de retorno futuras. Davidson (2002, p. 50) define esse axioma de forma precisa:

This ergodic axiom asserts, as a universal truth, that drawing a sample using past time-series and/or current cross-sectional market data is equivalent to drawing a sample from the universe of future market data. In an ergodic environment, the stochastic process generates immutable objective probabilities that govern all past, present and future data. Invoking the ergodic axiom means that the outcome at any future date is merely the statistical shadow of events that have occurred; the future is written in today's historical 'evidence'.

Se os mercados financeiros são eficientes e fundamentos de mercado imutáveis são os determinantes do retorno futuro dos ativos, então os agentes econômicos que cometem erros persistentemente (irrational noise traders), a 
explicação para a volatilidade observada no mercado financeiro, deveriam ser extintos via algum processo econômico Darwiniano ou eles sobreviveriam apenas se aprendessem como não cometerem erros persistentes, já que, no longo prazo, os preços dos ativos são determinados por seus valores fundamentais e inevitavelmente esses agentes seriam vencidos pelo mercado. Essa constatação leva Davidson (1998a) a afirmar que Stiglitz incorre em incoerência lógica ao reconhecer que a especulação no mercado financeiro não só persiste ao longo do tempo como aumenta. Desse modo, para explicar a persistência e aumento da especulação, Stiglitz apela para a autoridade:

To resolve this dilemma of the centuries old existence of speculation in financial markets, Stiglitz appeals to authority - the ultimate free market authority and successful circus impresario - P.T. Barnum. Stiglitz (1989, p. 106) misquotes Barnum's dictum 'There's a sucker born every minute. As 'There is a fool born every moment' and even incorrectly attributes this homily to one G. T. Barnum. Nevertheless, Stiglitz's appeal to Barnum's authority implies that society continues to produce, even in the long rum, fools who irrationally believe they can beat the market.

Faced with the contradiction between the implications of the efficient market hypothesis where those who make persistent errors are eradicated and his attribution of volatile financial markets to the persistent existence of foolish market participants, Stiglitz has done the only 'rational' thing that a potential Nobel prize recipient can do. He ignores this logical inconsistency (Davidson, 1998a, p. 5).

Mesmo apresentando um razoável conteúdo de crítica à abordagem convencional, as bases teóricas que fundamentam a análise de Stiglitz, se Davidson estiver correto, como acreditamos, não lhe permitem fornecer uma análise consistente acerca do comportamento do mercado financeiro do mundo real, de modo que "Stiglitz's problem is that he has confused the logic of efficient financial market behavior in a presumed ergodic system with real world financial market behavior when agents know they are dealing with an uncertain (nonergodic) future" (Davidson, 2002, p. 185).

Até essa etapa do trabalho, ainda no âmbito da abordagem convencional, ao mostrar posições conflitantes dentro da literatura teórica, a ausência de evidências econométricas robustas e a fragilidade dos argumentos históricos, procurou-se desenvolver uma abordagem crítica que permite questionar se são realmente robustas as teorias que estabelecem um conjunto de canais por meio dos quais a Integração Financeira Internacional estimularia o crescimento econômico de longo prazo dos países. Tendo como ponto de partida esse questionamento, o próximo passo é elaborar, ainda que de forma incipiente, uma crítica à abordagem convencional e hipóteses alternativas acerca da relação entre Integração Financeira Internacional e crescimento econômico, tendo por base fundamentos teóricos de inspiração (pós) Keynesiana acerca do funcionamento do mercado financeiro. 


\section{A crítica pós-Keynesiana}

A literatura de inspiração (pós) Keynesiana permite elaborar uma crítica fundamental, tendo como pressupostos teóricos hipóteses alternativas acerca do funcionamento do mercado financeiro. Nesta perspectiva, parte-se do pressuposto de existência de incerteza fundamental ou, na linguagem de Davidson (2002, p. 187), que o sistema econômico é não ergódico, onde o futuro é incerto no sentido de que o lucro atuarial ou uma expectativa de ganho baseada em cálculos matemáticos confiáveis, realizados de acordo com funções de probabilidade existentes, não pode ser obtida de algum conjunto de dados. Essa situação de incerteza que permeia as decisões dos agentes econômicos fica bastante clara na seguinte passagem:

Desejo explicitar que por conhecimento "incerto" não pretendo apenas distinguir o que é conhecido como certo, do que apenas é provável. [...] O sentido em que estou usando o termo é aquele segundo o qual a perspectiva de uma guerra européia é incerta, o mesmo ocorrendo com o preço do cobre e a taxa de juros daqui a vinte anos, ou a obsolescência de uma nova invenção, ou a posição dos proprietários particulares de riqueza no sistema social de 1970. Sobre esses problemas não existe qualquer base científica para um cálculo probabilístico. Simplesmente, nada sabemos a respeito (Keynes, 1937, p. 171).

Especificamente em relação aos mercados financeiros, como ressalta Carvalho (2004, p. 8), esse ambiente de incerteza é ainda mais importante, já que, na negociação de ativos, negocia-se, na verdade, recompensas futuras, a serem usufruídas sob condições que também só se revelarão no futuro. Não é razoável esperar que um mercado financeiro caracterizado pela existência de incerteza fundamental seja eficiente para alocar capital escasso entre usos alternativos, ou seja, revelar os valores fundamentais subjacentes aos ativos financeiros, pelo contrário, nessa perspectiva, a função do mercado financeiro não é alocar capital de forma eficiente, mas fornecer liquidez aos agentes econômicos. Isso fica claro na argumentação de Davidson (2002, p. 187):

[...] even if 'fundamentals' exist today and even if a data set permits one to estimate today's (presumed to exist) objective conditional probability distribution, such calculations do not form a reliable base for forecasting the future. Today's conditional objective probabilities are not reliable actuarial guides to the future.

As we have already noted, Keynes's description of uncertainty matches technically what mathematical statisticians call a nonergodic stochastic system. In a nonergodic system, one can never expect whatever data set exists today to provide a reliable guide to future outcomes. In such world, markets cannot be efficient. Instead the primary function of financial markets is to provide liquidity.

Ao assumir a existência da incerteza fundamental, portanto um sistema econômico não ergódico, rejeita-se a hipótese de mercados eficientes, pressuposto teórico subjacente aos argumentos segundo os quais a Integração Financeira 
Internacional potencialmente estimularia o crescimento econômico de longo prazo dos países. Ao rejeitar o pressuposto de mercados eficientes como adequado para explicar o funcionamento do mercado financeiro internacional do mundo real, fazse uma crítica mais contundente aos proponentes do livre fluxo de capitais entre as fronteiras do que a crítica presente na assunção de assimetrias de informação nos contratos financeiros. Se a função do mercado financeiro não é alocar capital de forma eficiente, então não existe razão para esperar que a Integração Financeira Internacional levará a maior crescimento econômico, nem no curto nem no longo prazo. Davidson (1998b, p. 2) argumenta especificamente a esse respeito:

The mantra of EMT (Efficient Market Theory) is 'the market knows best' how to optimally allocate scarce capital resources and promote maximum economic growth.

Since the 1970s, this 'compelling' efficient market logic has provided the justification for nations to dismantle most of the post-war capital regulation in financial markets. The argument for this 'liberalization' of financial markets was that it would produce lower real costs of capital and higher output and productivity growth rates compared to the growth rates experienced between World War II and 1973 when international capital flow controls were practiced by most countries of the world, including the United States.

In Keynes's General Theory the primary function of financial markets is to provided liquidity not efficiency, consequently real world international capital markets can never deliver, in either the short-run or the long-run, the results claimed by EMT.

Davidson (2003, p. 7) conclui que "Instead of producing the utopian promises of greater stability and more rapid economic growth promised by classical economists, liberalization of capital flow regulations has been associated with exchange rate instability, slower global economic growth, and higher global unemployment [...]". Essa instabilidade na taxa de câmbio tende a deprimir a taxa global de investimento pelos seguintes motivos:

i) significativas mudanças na taxa de câmbio afetam a posição de competitividade internacional das indústrias domésticas vis-à-vis indústrias externas e, portanto, tende a diminuir os incentivos para investimento em grandes projetos com custos afundados irreversíveis;

ii) em um mundo incerto onde o futuro não pode ser previsto confiavelmente de sinais de preços passados e presentes, a volatilidade da taxa de câmbio inviabiliza a confiança dos empresários na habilidade deles para avaliarem o potencial de lucratividade de algum projeto grande de investimento;

iii) todo aumento na taxa de câmbio não ameaça somente as indústrias domésticas com perdas de mercados para exportação, mas também perda de participação no mercado interno, quando as importações tornam-se mais baratas. 
Em um ambiente de incerteza, os agentes econômicos adotam o que Keynes $(1936,1937)$ chama de guia prático do futuro - convenções: considerar a situação atual, projetando-a para o futuro e só mudando quando se tem razões mais ou menos claras para esperar alguma mudança; tomar decisões tendo por base a opinião da média ou maioria, ou seja, a chamada psicologia das massas. Além da precariedade desse método, Keynes (1936, p. 113) lista alguns fatores que podem agravar ainda mais essa precariedade e, portanto, elevar a instabilidade do sistema:

i) o fato de grande parte dos participantes do mercado financeiro não possuírem conhecimento razoável das circunstâncias, diminuindo o elemento de conhecimento real das avaliações;

ii) o fato de uma avaliação convencional baseada na psicologia das massas apresentar grande sensibilidade em relação a mudanças repentinas na opinião;

iii) o comportamento de investidores profissionais e especuladores, que consiste em antecipar qual será o estado de opinião do mercado num futuro próximo. Ou seja, possibilidade de prever a opinião da média, podendo resultar na obtenção de lucros por conta de valorizações ou desvalorizações repentinas nos preços de títulos e ações.

Eatwell (1996, p. 35) argumenta que essa caracterização feita por Keynes acerca da operação do mercado financeiro sugere dois canais por meio dos quais a Integração Financeira Internacional pode resultar em uma deterioração da performance econômica:

i) primeiro, um mercado que opera como um 'concurso de beleza' é provável que seja altamente instável e propenso a severas perdas ocasionais de liquidez quando toda a opinião tende a mudar em uma mesma direção, o que aumentará os custos do capital e pode levar a severas escassezes de capital ambos fatores tenderão a desestimular investimentos e reduzir o nível de atividade no médio prazo;

ii) o funcionamento do mercado financeiro como um 'concurso de beleza', em um ambiente liberalizado, pode produzir mudanças no comportamento de governantes, ao tornar necessária a manutenção da 'credibilidade de mercado'. Isso significa que governantes podem ser forçados a seguirem políticas preferidas pelo mercado financeiro, incompatíveis com uma taxa de investimento e crescimento desejáveis. A esse respeito:

In Keynes's characterization of the operations of a market economy, it is clear that the behaviour of financial markets may well be an important factor driving the economy towards a low-growth, high-unemployment equilibrium. The markets are neither omniscient nor benign. When their influence is combined with the persistent search for government 'credibility', defined in terms of 'sound money' and 'prudent' deflationary policies, then the low level position is the most likely outcome (Eatwell, 1996, p. 39). 
Portanto, as relações entre Integração Financeira Internacional e crescimento econômico dependem sobremaneira dos fundamentos teóricos aceitos como plausíveis para explicar o comportamento do mercado financeiro internacional. Essa perspectiva (pós) Keynesiana fornece uma interpretação alternativa à combinação ortodoxa de Economia do Bem-Estar e Hipótese de Mercados Eficientes.

\section{Considerações finais}

Se no âmbito teórico existem opiniões divergentes dentro da literatura convencional quanto aos potenciais benefícios resultantes da Integração Financeira Internacional, cabendo destaque à abordagem crítica apresentada por Stiglitz (2000, 2003), quem apresenta argumentos para fundamentar a hipótese de que a Integração Financeira Internacional teria efeitos negativos sobre o crescimento econômico dos países, os trabalhos empíricos são ainda menos conclusivos. Não existem evidências empíricas suficientemente robustas para corroborarem a hipótese de que a Integração Financeira Internacional estimula o crescimento econômico de longo prazo, tanto nos países desenvolvidos quanto em desenvolvimento, e mesmo considerando que essa relação depende da existência prévia de certas condições, como desenvolvimento institucional dos países, desenvolvimento do sistema financeiro doméstico, grau de abertura das economias ao comércio internacional e prática de políticas econômicas consideradas 'sadias' pela literatura convencional.

Uma crítica a essa visão oferece hipóteses alternativas acerca da relação entre Integração Financeira Internacional e crescimento econômico. Nesta visão alternativa de inspiração (pós) Keynesiana, os agentes econômicos tomam decisões em um ambiente caracterizado por incerteza fundamental (um sistema não ergódico), adotando um conjunto de 'regras práticas' em relação ao futuro (convenções). Nessa perspectiva, a função do mercado financeiro é fornecer liquidez e não alocar capital de forma eficiente entre usos alternativos, de modo que não é razoável esperar, tanto no curto quanto no longo prazo, que a Integração Financeira Internacional estimule o crescimento econômico. No entanto, nessa perspectiva teórica alternativa, não obstante o esforço de pesquisa empreendido por vários autores, as análises não apresentam uma abordagem sistemática acerca das possíveis conexões entre Integração Financeira Internacional e crescimento econômico, o que levou Carvalho (2004, p. 14) a afirmar que uma "área promissora para pesquisas é o mapeamento dos canais pelos quais controles de capitais influenciam [...] as metas últimas da sociedade, como o crescimento e o pleno-emprego". 


\section{Referências bibliográficas}

AGÉNOR, Pierre-Richard. Benefits and costs of international financial integration: theory and facts. Washington, DC: The World Bank, 2003.

ALVES JR., A. J.; FERRARI FILHO, F.; PAULA, L. F. The post Keynesian critique of conventional currency crisis models and Davidson's proposal to reform the international monetary System. Journal of Post Keynesian Economics, v. 22, n. 2, p. 209-227, Winter, 1999-2000.

BAILLIU, J. N. Private capital flows, financial development, and economic growth in developing countries. Ottawa: Bank of Canada, 2000. (Working Paper).

BOORMAN, J.; ALLEN, M. A new framework for private sector involvement in crisis prevention and crisis management. In: REFORMING the International Financial System: Crisis Prevention and Response, FONDAD, Dec. 2000.

CARKOVIC, M.; LEVINE, R. Does foreign direct investment accelerate economic growth? University of Minnesota, 2002.

CARVALHO, Fernando J. Cardim de. Controles de capitais: uma agenda de pesquisa. Rio de Janeiro: IE-UFRJ, Grupo Moeda, 2004. (Texto para Discussão).

et al. Controvérsias recentes sobre controles de capitais. Rio de Janeiro: IEUFRJ, Grupo Moeda, 2003. (não publicado).

; SICSÚ, J. Teorias e experiências de controles do fluxo de capitais: focando o caso da Malásia. Rio de Janeiro: IE-UFRJ, 2004. (Texto para Discussão).

COOPER, R. N. Should capital-account convertibility be a world objective? In: PETER, B. Kenen (Ed.). Should the IMF pursue capital-account convertibility. Princeton University, Department of Economics, 1998. (Essays in International Finance, n. 207).

DAVIDSON, P. Volatile financial markets and the speculator. Paper presented as The Economic Issues Lecture to the Royal Economic Society Annual Conference, Warwick, England, Apr. 1998a.

The case for regulating international capital flows. Paper presented at the Social Market Foundation Seminar on Regulation of Capital Movements, London, Nov. 17, 1998b.

2002.

Financial markets, money and the real world. Massachusetts: Edward Elgar,

The future of the International Financial System. Paper presented at Conference on the Future of Economics at Cambridge University, Sept. 18, 2003.

DEVLIN, Robert; et al. Crescimento dos fluxos de capital e desenvolvimento: uma visão geral das questões de política econômica. Pesquisa e Planejamento Econômico, Rio de Janeiro, v. 24, n. 3, p. 411-444, 1994.

EATWELL, John. International financial liberalization: the impact on world development. New York: CEPA, 1996. (Working Paper, n. 1). 
EDISON, H. J.; KLEIN, M. W.; RICCI, L.; SLOEK, T. Capital account liberalization and economic performance: survey and synthesis. Massachusetts: National Bureau of Economic Research, 2002. (Working Paper, n. 9100).

; LEVINE, R.; RICCI, L.; SLOK, T. International financial integration and economic growth. Massachusetts: National Bureau of Economic Research, 2002. (Working Paper, n. 9164).

EICHENGREEN, B. et al. Capital account liberalization: theoretical and practical aspects. Washington, DC: International Monetary Fund, 1998. (Occasional Paper, n. 172).

FISHER, Stanley. Capital account liberalization and the role of the IMF. In: PETER, B. Kenen (Ed.). Should the IMF pursue capital-account convertibility. Princeton University, Department of Economics, 1998. (Essays in International Finance, n. 207).

KLEIN, M.; OLIVEI, G. Capital account liberalization, financial depth, and economic growth. Massachusetts, National Bureau of Economic Research, 1999. (Working Paper, n. 7384).

McLEAN, B.; SHRESTHA, S. International financial liberalization and economic growth. Sydney: Reserve Bank of Australia, 2002. (Research Discussion Paper, n. 3).

MISHKIN, F. Understanding Financial Crises: A Developing Country Perspective. Massachusetts, National Bureau of Economic Research, 1996. (Working Paper, n. 5600).

OBSTFELD, M. Risk-taking, global diversification and growth. American Economic Review, v. 84, n. 5, p. 1310-1329, 1994.

PRASAD, E.; ROGOFF, K. et al. Effects of financial globalization on developing countries: some empirical evidence. International Monetary Found, 2003.

QUINN, D. The correlates of change in international financial regulation. American Political Science Review, v. 91, n. 3, p. 531-551, 1997.

REISEN, H. et al. Which types of capital inflows foster developing-country growth. International Finance, v. 4, n. 1, p. 1-14, 2001.

RODRIK, D. Who needs capital-account convertibility? In: PETER, B. K. (Ed.). Should the IMF pursue capital-account convertibility. Princeton University, Department of Economics, 1998. (Essays in International Finance, n. 207).

ROGOFF, K. Managing the world economy. The Economist, Aug. 3, 2002.

SOTO, M. Capital flows and growth in developing countries: recent empirical evidence. Paris: OECD Development Centre, 2000. (Technical Papers, n. 160).

STIGLITZ, J. Capital market liberalization, economic growth, and instability. World Development, Washington, DC, World Bank, v. 28, n. 6, p. 1075-1086, 2000.

Globalization and growth in emerging markets and the new economy. Journal of Policy Modeling, v. 25, p. 505-524, 2003. 